\title{
BANANA AND PLANTAIN (Musa spp.) CULTIVAR PREFERENCE, LOCAL PROCESSING TECHNIQUES AND CONSUMPTION PATTERNS IN EASTERN DEMOCRATIC REPUBLIC OF CONGO
}

\author{
EKESA B.N. ${ }^{*}$, KIMIYWE J.2, DAVEY M.W. ${ }^{3}$, DHUIQUE-MAYER C. ${ }^{4}$, VAN DEN BERGH I. ${ }^{5}$ KARAMURA D. ${ }^{1}$ AND \\ BLOMME G. ${ }^{1}$
}

1Bioversity International, Plot 106, Katalima Road, P.O. Box 24384, Kampala, Uganda.

${ }^{2}$ Kenyatta University, Department of Food, Nutrition and Dietetics, P.O. Box 43844, Nairobi, Kenya.

${ }^{3}$ Lab Fruit Breeding and Biotechnology, Department of Biosystems, Katholieke University of Leuven (KUL), de Croylaan 42, Heverlee, B-3000, Leuven, Belgium.

${ }^{4}$ Centre de Coopération Internationale en Recherche Agronomique pour le Développement (CIRAD), Dept. Persyst-UMR Qualisud TA B95/16 73 av. J.F Breton 34398 Montpellier Cedex 5, France.

${ }^{5}$ Bioversity International, Parc Scientifique Agropolis II 34397 Montpellier Cedex 5, France.

*Corresponding Author: Email- b.ekesa@cgiar.org

Received: November 02, 2012; Accepted: December 03, 2012

\begin{abstract}
This cross-sectional study carried out in North Kivu (NK) and South Kivu (SK) of the Eastern Democratic Republic of Congo aimed to identify the most preferred Musa cultivars, their processing/cooking methods and Musa consumption patterns among rural households. Our results showed that the preferred cooking banana varieties included yellow-pulped AAA-East African Highland bananas [EAHB] 'Nshikazi' (SK) and 'Vulambya' (NK), which were valued for their cooking qualities, large bunches and suitability for production of banana beer. The preferred plantains were orange-pulped and included 'Musheba' (SK) and 'Musilongo' (NK) and were preferred for their short maturation period, large bunches and higher market prices. Over $60 \%$ of the households examined had consumed EAHB within 24 hours, whereas $<10 \%$ had consumed plantains. The most common cooking method was simple boiling of bananas/plantains and main accompaniments include beans and amaranth leaves. Over $69 \%$ of surveyed households that had consumed Musa products had consumed them boiled. The majority of the households ( $90 \%$ ) obtained banana/plantains from their farms, and $>55 \%$ of households from SK and NK consumed banana products between 2 and 4 times/week. This information will be used to help direct researchers on the Musa cultivars that could be further sampled for carotenoid analysis and those found to be rich could be used in the fast-tracking approach to reduce Vitamin A deficiency.
\end{abstract}

Keywords- Banana, plantain, preference, cooking, Democratic Republic of Congo (DRC)

Citation: Ekesa B.N., et al (2012) Banana and Plantain (Musa spp.) Cultivar Preference, Local Processing Techniques and Consumption Patterns in Eastern Democratic Republic of Congo. International Journal of Agriculture Sciences, ISSN: 0975-3710 \& E-ISSN: 0975-9107, Volume 4, Issue 8, pp-312-319.

Copyright: Copyright@2012 Ekesa B.N., et al. This is an open-access article distributed under the terms of the Creative Commons Attribution License, which permits unrestricted use, distribution and reproduction in any medium, provided the original author and source are credited.

\section{Introduction}

Plantain and bananas (Musa spp.) serve as important food crops in much of Africa. Together they provide over $25 \%$ of the carbohydrate needs and $10 \%$ of the daily calorie requirements for over 70 million people on the African continent [1]. Production of the plantains and bananas is concentrated in Eastern DRC and ranges between 75,000 and 80,000 tonnes per year, and they rank second in importance after cassava in the Democratic Republic of Congo (DRC) [2]. The majority of cultivated bananas and plantains are triploid varieties belonging to the Eumusa section of the genus Musa, family Musaceae [3]. These varieties evolved from intra- and inter-specific crosses involving two diploid ancestor species, $M$. acuminata Colla (genome AA) and M. balbisiana Colla (genome $\mathrm{BB})$, which originated from Malaysia and India, respectively [4].
Bananas and plantains are one of the main dietary starchy sources [2], apart from this, studies have suggested that some cultivars have high pro-vitamin A carotenoids (pVACs) levels and are capable of providing up to half of the total human daily vitamin A requirement in a single fruit [5,6]. PVACs among cultivars of Musa fruits differ more than 200-fold, ranging from $0.23-59.56 \mu \mathrm{g} / \mathrm{gdw}$, with very high levels found in plantains and lower levels in other cooking bananas and commercial dessert types [1]. PVAC levels are linked with orange fruit pulp color, with most dessert bananas having a light colored pulp (cream, ivory) and most plantain cultivars having more yellow-orange colored pulp [1].

Although there is no documentation on the prevalence of vitamin $\mathrm{A}$ deficiency (VAD) in Esstern DRC, based on WHO estimates, $61.1 \%$ of preschool children in the whole of DRC have VAD. AC- 
cording to the classification provided by the International vitamin A Consultative Group (known as the Annecy Accords), a prevalence of over $30 \%$ of the population with less than $20 \mu \mathrm{g} / \mathrm{dl}$ serum retinol defines a situation of severe VAD [7]. In addition, DRC has one of the highest under-five mortality rates (U5MR) in the world, with more than 200 deaths in every 1000 live births [2]. The most obvious result of VAD is progressive damage to the eyes, eventually leading to blindness. Sub-clinical VAD is a much more widespread problem, contributing to an increased risk of morbidity and mortality from common childhood infections [8]. Therefore, with Musa fruit consumption levels ranging from 137-to $174 \mathrm{~kg} /$ person/year in DRC [9], it is clear that Musa fruit could be an important source of carotenoids and other micronutrients to consumers.

Apart from the high banana and plantain production, DRC is endowed with a wealth of natural resources and has a substantial agricultural potential, capable of feeding the whole of Africa [10]. Unfortunately this potential has not been exploited to improve living standards for the general Congolese population [10]. Out of the 154 territories (districts) in the whole of DRC, more than 117 are moderately food insecure and 28 others are in a state of acute food insecurity and livelihood crisis. Most of the food-insecure households are concentrated in the eastern part of DRC and more specifically in North and South Kivu [10].

Despite the major role that bananas and plantains play in Eastern DRC, there has been limited research and hardly any documentation on the preferences for the various banana cultivars grown, their processing/cooking techniques and consumption patterns among small-holder farmer households. In addition, the nutrition value of the preferred Musa cultivars and in particular pVAC levels have yet to be determined to establish whether they could be used in approaches aimed at reducing VAD among households.

\section{Methodology}

A cross-sectional study was carried out in Eastern DRC using focus group discussions (FGDs) and household interviews (HHIs), during the month of September 2009. Two provinces, North Kivu (NK) and South Kivu (SK), were selected based on the high levels of food insecurity and their high dependency on bananas and plantains [11]. Multistage sampling was used to select one territory from each of the provinces and three localities from each of the two territories. Two territories/districts Beni and Bukavu were sampled from North Kivu and South Kivu, respectively. In Beni territory Mabuku, Kisungu and Rwakhwa localities were randomly sampled and in Bukavu territory, the localities randomly sampled were Kajeje, Murhesa and Miti. A listing of all households with preschool children in each locality was compiled and systematic random sampling was used to select the specific households to be interviewed. Sample size was calculated using Fisher's formula:

$$
n=\frac{t^{2} \times p(1-p)}{m^{2}}
$$

where $\mathrm{n}=$ required sample size, $\mathrm{t}=$ confidence level at $95 \%$ (standard value of 1.96), $p=$ estimated proportion of households with preschool children with regards to total households and $\mathrm{m}=$ margin of error at $5 \%$ (standard value of 0.05 ) [12]. This gave a sample size of 163 and 208 households with preschool children from Beni territory and Bukavu territory, respectively. Focus group discussions were carried out at the locality level, to obtain information on the most popular and preferred Musa cultivars within the community and the general processing (e.g., cooking) methods. Information obtained through FGDs was further verified using household interviews by which structured questionnaires were administered to mothers (caregivers) of children below 5 years old. FAO studies confirm that rural women make a tremendous contribution to food and agricultural production. They also play a crucial role in determining and guaranteeing food security and well-being for the entire household [13], it was therefore necessary that questions requiring details on household consumption patterns were addressed to women (caregivers); women respondents were purposefully selected for the household interviews.

Apart from verification, household interviews were also used to collect information on household food consumption patterns with regards to bananas and plantains. A ranking system based on traits identified as important to the community was used to establish the preference level among the different Musa cultivars. After identification of the two most preferred cultivars from each site (NK and SK), agronomists working on banana research identified mature plants of each of the popular cultivars from farmer fields and marked them. A mature bunch was harvested and fruit samples obtained using standardized sampling techniques where two fruits were obtained from the middle hand of the bunch at ripening stage 1 (hard and green) [14]. Pre-screening of fruit pulp color for carotenoid content was carried out using a standardized color strip developed by HarvestPlus reflecting the total carotenoid content of starchy staples. Descriptive statistics were computed using the statistical package for social sciences (SPSS) package and data were summarized in frequency tables, charts and graphs.

\section{Results}

\section{Demographic Data}

Of the 371 respondents from SK $(\mathrm{N}=208)$ and NK $(\mathrm{N}=163), 92 \%$ were women, $83 \%$ were monogamously married. Forty seven percent of the respondents from both NK and SK were aged between 25-34 years [Fig-1] and the youngest respondent was 15 years old.

The majority of the households $(61 \% \mathrm{~N}=371)$ consisted of 5 to 8 members with an average household size of 6.4 members in NK and 7.1 in SK. Forty five percent of the respondents had not received any form of formal education, $32 \%$ had received incomplete primary education, $11 \%$ had completed primary education, while only $0.5 \%$ had gone on to a vocational college and none of the respondent had any college or university diploma/degree. Most of the households $(76 \%)$ interviewed depended on agricultural production as the main source of income, $14 \%$ were involved in casual labor, while $5.3 \%$ and $4.3 \%$ were involved in small-scale business and formal employment, respectively. The mean monthly household income was $\$$ US20-50 per month, but over $80 \%$ of the households had a monthly average income of less than \$US30.

\section{Most Popular and Preferred Musa Cultivars}

Through 14 FGDs in SK(8) and NK (6), a list of all Musa cultivars grown within the community was compiled. A total of 16 and 19 Musa cultivars were listed in SK and NK, respectively. The participants then arranged the named cultivars in order of priority. [Table1] gives the physiological descriptions, synonyms and botanic char- 
acteristics used in the identification of the four most popular Musa cultivars in SK, Bukavu territory and NK, Beni territory identified by participants of the FGDs. In SK, Bukavu territory, the four most popular cultivars consisted of three East African Highland cooking banana (AAA-EAHB) cultivars and one plantain ( $A A B)$ cultivar while in NK, Beni territory the four included two East African Highland cooking banana (AAA-EAHB) cultivars and two plantain $(A A B)$ cultivars. The cultivars were then scored on a scale of 1 to 5 by FGD participants for cooking quality, market price, bunch size (yield) and plant disease resistance in order to identify the two most popular and preferred from each of the two sites. In SK, the EAHB 'Nshikazi' also called 'Magizi' had the best overall score, with 4.65 for cooking quality, 3.93 for market price, 4.02 for bunch size and 2.39 for disease resistance [Table-2]. The second highest score was obtained by the plantain 'Musheba', with 3.98 for cooking, 4.60 for market price, 3.43 for bunch size (yield) and 2.08 for disease resistance. In NK, the EAHB 'Vulambya' received the highest score, followed by the plantain 'Musilongo', the traits that made 'Vulambya' and 'Musheba' popular were similar to those observed in 'Nshikazi' and 'Musheba' respectively from SK. The principal weakness of the popular cultivars was their low disease resistance [Table-2].

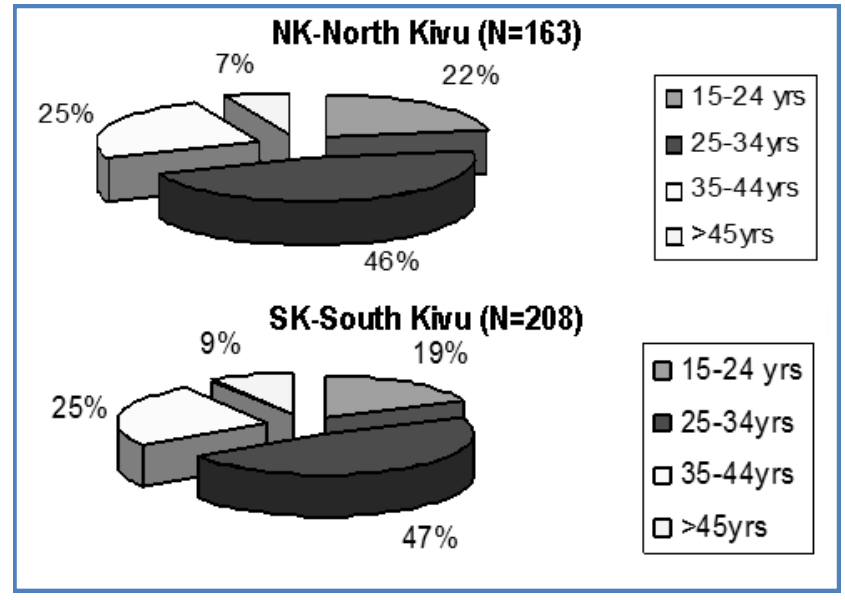

Fig. 1- Age structure of survey respondents in Eastern Democratic Republic of Congo

Table 1- Description and Botanic characteristics used in identification of four most popular cultivars from Beni territory (North Kivu) and Bukavu territory (South Kivu)

\begin{tabular}{|c|c|c|c|c|c|c|c|c|c|}
\hline \multirow{2}{*}{ Local name } & \multirow{2}{*}{ Origin } & \multirow{2}{*}{ Synonyms } & \multirow{2}{*}{$\begin{array}{l}\text { Genomic } \\
\text { group }\end{array}$} & \multirow{2}{*}{ Sub-group } & \multicolumn{5}{|c|}{ Botanic characteristic used to identify the cultivar } \\
\hline & & & & & Pseudostem & leaves & Bunch & Fruit & Male bud \\
\hline Vulambya & Beni (DRC) & $\begin{array}{c}\text { Malambya }^{2} \text {, } \\
\text { Bulambya }^{2}, \\
\text { Nyalambya }^{2}, \\
\text { Nyalambi² (Rukonjo } \\
\text { (Uganda) }^{3}\end{array}$ & AAA-EA2 & Lujugira-Mutika² & $\begin{array}{l}\geq 3 \text { metres light } \\
\text { green with } \\
\text { black blotches }{ }^{2}\end{array}$ & Dirty green² & $\begin{array}{l}\text { Hangs vertically, } \\
\text { Compact, } \\
\text { cylindrical, } \\
\text { maximum } 11 \text { hands² }\end{array}$ & $\begin{array}{l}\text { Straight, Medium } \\
(15 \mathrm{~cm}-20 \mathrm{~cm} \text { long), } \\
\text { pointed tips, maxi- } \\
\text { mum } 18 \text { fruits in } 3 \mathrm{rd} \\
\text { hand, naked tips }{ }^{2}\end{array}$ & $\begin{array}{c}\text { Ovoid shaped, at } \\
\text { harvest about } \\
23 \mathrm{~cm} \text { width and } \\
36 \mathrm{~cm} \text { length, white } \\
\text { male flower }{ }^{2}\end{array}$ \\
\hline Musilongo & Beni (DRC) & $\begin{array}{c}\text { Kasilongo }^{2} \\
\text { Munzabo }^{2} \\
\text { Kibeda }\end{array}$ & $\mathrm{AAB}^{2,4}$ & $\begin{array}{c}\text { Plantain } \\
\text { (French horn })^{2}\end{array}$ & $\begin{array}{c}\geq 3 \text { metres } \\
\text { Red-purple }^{2}\end{array}$ & Medium green², & $\begin{array}{l}\text { Hangs vertically, } \\
\text { Lax, } \\
\text { assymetrical, } \\
\text { maximum number of } \\
\text { hands } 9^{2}\end{array}$ & $\begin{array}{l}\text { Sharp curved long } \\
\text { fruit, pointed tip } \\
\text { about } 14 \text { fruits in } \\
\text { 3rd hand, persistent } \\
\text { floral remains on } \\
\text { fruit tips } 2\end{array}$ & $\begin{array}{c}\text { Lanceolate } \\
\text { shaped, yellow } \\
\text { male flower }{ }^{2}\end{array}$ \\
\hline Nguma & Beni (DRC) & $\begin{array}{l}\text { Mbaguma2 }^{2} \\
\text { Apakumo }^{2} \\
\text { Asongbe }^{2}\end{array}$ & $\mathrm{AAB} 24$ & $\begin{array}{c}\text { Plantain } \\
\text { (French horn) }{ }^{2}\end{array}$ & $\begin{array}{c}\geq 3 \text { metres } \\
\text { Light Green } \\
\text { almost yellow- } \\
\text { ish }^{2}\end{array}$ & Medium green ${ }^{2}$ & $\begin{array}{l}\text { Hangs vertically, } \\
\text { lax, } \\
\text { slightly truncated } \\
\text { cone shape }\end{array}$ & $\begin{array}{c}\text { Fruits almost } \\
\text { straight and a bit } \\
\text { inflated. Slightly } \\
\text { pointed tips with } \\
\text { persistent old style } 2\end{array}$ & $\begin{array}{l}\text { Ovoid, large, red } \\
\text { purple and persis- } \\
\text { tent, yellow male } \\
\text { flower }{ }^{2}\end{array}$ \\
\hline Kiware & Beni (DRC) & $\begin{array}{c}\text { Ndabaware }^{2}, \\
\text { Maware }^{2} \\
\text { Ndyabawali2 } \\
\text { Ngagara }(\mathrm{Ug})^{3} \\
\text { Nandigobe }(\mathrm{Ug})^{3}\end{array}$ & AAA-EA ${ }^{2}$ & Lujugira-Mutika² & $\begin{array}{l}\quad \geq 3 \text { metres } \\
\text { light green with } \\
\text { black blotches }{ }^{2}\end{array}$ & Dirty green ${ }^{2}$ & $\begin{array}{l}\text { Hanging vertically, } \\
\text { cylindrical, } \\
\text { lax. Rachis with } \\
\text { semi persistent } \\
\text { floral parts, } \\
10 \text { hands }\end{array}$ & $\begin{array}{l}\text { Fruits slender and } \\
\text { angular slightly } \\
\text { above } 20 \mathrm{~cm} \text {, with } \\
\text { persitent style on } \\
\text { the fruit tip, } 18 \text { fruits } \\
\text { in } 3^{\text {rd }} \text { hand } 2\end{array}$ & $\begin{array}{l}\text { Intermediate Pur- } \\
\text { ple and pointed, } \\
\text { yellow make flow- } \\
\text { er }^{2}\end{array}$ \\
\hline Nshikazi & $\begin{array}{l}\text { Bukavu } \\
\text { (DRC) }\end{array}$ & $\begin{array}{l}\text { 'Magizi'= "bitter" }{ }^{3} \\
\text { Ishika'(Rwanda), }{ }^{3} \\
\text { Ensika',(Ug) }{ }^{3} \\
\text { 'Emburansika' } \\
\text { Omuburasika'(Ug33 }\end{array}$ & AAA-EA 4 & Lujugira-Mutika & $\begin{array}{l}\quad \geq 3 \text { metres } \\
\text { light green with } \\
\text { black blotches }{ }^{2}\end{array}$ & Dirty green ${ }^{2}$ & $\begin{array}{c}\text { Oblique and com- } \\
\text { pact cylindrical } \\
\text { bunch }^{2}\end{array}$ & $\begin{array}{l}\text { Fruits medium and } \\
\text { pulp astringent }{ }^{2}\end{array}$ & $\begin{array}{l}\text { Ovoid shaped, at } \\
\text { harvest about } \\
23 \mathrm{~cm} \text { width and } \\
36 \mathrm{~cm} \text { length, white } \\
\text { male flower }{ }^{2}\end{array}$ \\
\hline Musheba & $\begin{array}{l}\text { Bukavu } \\
\text { (DRC) }\end{array}$ & $\begin{array}{l}\text { Busheba }{ }^{3} \text {, } \\
\text { misheba }\end{array}$ & $\mathrm{AAB}^{4}$ & $\begin{array}{l}\text { Plantain } \\
\text { (French) }\end{array}$ & $\begin{array}{c}\geq 3 \text { metres } \\
\text { Light green, } \\
\text { almost yellow- } \\
\text { ish }{ }^{2}\end{array}$ & Medium green ${ }^{2}$ & $\begin{array}{l}\text { Hangs vertically, } \\
\text { lax, } \\
\text { slightly truncated } \\
\text { cone shape }{ }^{2}\end{array}$ & $\begin{array}{c}\text { Fruits almost } \\
\text { straight and a bit } \\
\text { inflated. Slightly } \\
\text { pointed tips with } \\
\text { persistent old style² }\end{array}$ & $\begin{array}{c}\text { Male bud large, red } \\
\text { purple and persis- } \\
\text { tent, yellow male } \\
\text { flower }{ }^{2}\end{array}$ \\
\hline Barhebesha & $\begin{array}{l}\text { Bukavu } \\
\text { (DRC) }\end{array}$ & $\begin{array}{c}\text { Njakara, } \\
\text { Incakara, } \\
\text { Barabesha } \\
\text { Mutant of mudjuva }\end{array}$ & AAA-EA 4 & Lujugira-Mutika² & $\begin{array}{c}\geq 3 \text { metres } \\
\text { Green with } \\
\text { black blotches }{ }^{2}\end{array}$ & Dirty green ${ }^{2}$ & $\begin{array}{c}\text { Hangs vertically, } \\
\text { lax, } \\
\text { Almost cylindrical2 }\end{array}$ & $\begin{array}{c}\text { Fruits }>25 \mathrm{~cm}, \\
\text { almost curved } \\
\text { towards the rachis, } \\
\text { bottle necked tips }{ }^{2}\end{array}$ & $\begin{array}{c}\text { Intermediate, } \\
\text { Purple, pointed, } \\
\text { white male flower }{ }^{2}\end{array}$ \\
\hline Kamera & $\begin{array}{l}\text { Bukavu } \\
\text { (DRC) }\end{array}$ & $\begin{array}{c}\text { Kamaramasenge, } \\
\text { Kilore }^{2}\end{array}$ & $\mathrm{AAB} 2,4$ & $\begin{array}{c}\text { Kamaramaseng } \\
\mathrm{e}^{2}\end{array}$ & $\begin{array}{l}\text { Green yellow } \\
\text { with bron rusty } \\
\text { pigmentation }\end{array}$ & Medium green² & $\begin{array}{c}\text { Asymmetrical-bunch } \\
\text { axis nearly straight, } \\
\text { compact, with bare } \\
\text { rachis }^{2}\end{array}$ & $\begin{array}{l}\text { Short } 15 \mathrm{~cm} \text {, almost } \\
\text { perpendicular to } \\
\text { rachis } 2\end{array}$ & $\begin{array}{l}\text { Purplish blue, } \\
\text { imbricated and } \\
\text { pointed2[1] }\end{array}$ \\
\hline
\end{tabular}


Table 2- Most popular and preferred Musa cultivars grown and consumed by households in North Kivu-NK $(N=163)$ and South Kivu-SK (N=208), Eastern Democratic Republic of Congo.

\begin{tabular}{|c|c|c|c|c|c|c|c|c|}
\hline Cultivar & \multicolumn{8}{|c|}{$\begin{array}{c}\text { Trait/Attribute ( } 1=\text { lowest score and } 5=\text { highest score) } \\
\text { and standard deviation (S.D) }\end{array}$} \\
\hline $\begin{array}{l}\text { Name } \\
\text { (genome group) }\end{array}$ & Yield & S.D & $\begin{array}{l}\text { Market } \\
\text { price } \\
\text { South }\end{array}$ & S.D & $\begin{array}{l}\text { Cooking } \\
\text { quality } \\
\text { Region }\end{array}$ & S.D & $\begin{array}{l}\text { Disease } \\
\text { resistance }\end{array}$ & S.D $\begin{array}{l}\text { Total } \\
\text { Score }\end{array}$ \\
\hline Vshikazi (AAA) & 4.02 & 0.68 & 3.93 & 0.67 & 4.65 & 0.49 & 2.39 & 0.57 \\
\hline AA) & 3.46 & 0.77 & $7 \quad 2.44$ & 0.58 & 3.83 & 0.69 & & 0.5911 \\
\hline Kam & 2.2 & $0.6 \mathrm{~s}$ & 92.24 & 0.73 & 1.4 & 0.58 & 9 & $0.55 \quad 7.25$ \\
\hline Musl & 3.43 & 0.81 & 14.6 & 0.53 & 3.98 & 0.75 & 2.08 & $0.91 \quad 4.08$ \\
\hline \multicolumn{9}{|c|}{ North Kivu Region } \\
\hline Vula & 4.02 & 0.68 & 84.03 & 0.71 & 4.66 & 0.49 & 2.41 & 0.5715 \\
\hline Kiw & 3.35 & 0.74 & 42.71 & 0.69 & 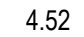 & 0.71 & & 0.6512 \\
\hline Ngur & 2.86 & 1.02 & $2 \quad 3.9$ & 0.75 & 2.32 & 0.79 & 2. & 0.6911 .65 \\
\hline Musilongo (AAB) & 3.39 & 0.93 & 34.63 & 0.52 & 3.86 & 0.73 & 2.12 & $0.91 \quad 14$ \\
\hline
\end{tabular}

The results regarding the preferred cultivars obtained through FGDs were verified during the individual household interviews. Respondents confirmed that 'Nshikazi' and 'Musheba' in South Kivu and 'Vulambya' and 'Musilongo' in North Kivu were the most preferred Musa cultivars [Table-3]. In SK more than half of the households (69\%) indicated 'Nshikazi' as the most preferred cultivar. The only popular and preferred plantain was 'Musheba'. In NK, 'Vulambya' was the most preferred cultivar overall (36\%). The two popular plantain cultivars were; 'Nguma' and 'Musilongo' although more households preferred 'Musilongo' to 'Nguma' [Table-3]. [Table-3] also shows that the most preferred cultivars had pulp color ranging from ivory and yellow (EAHB cooking varieties) to orange (plantain varieties).

Table 3- Musa cultivars preferred by household members in North Kivu ( $N=163)$ and South Kivu ( $N=208)$, Eastern Democratic Republic of Congo

\begin{tabular}{|c|c|c|c|c|c|}
\hline \multirow[t]{2}{*}{$\begin{array}{l}\text { Cultivar local } \\
\text { Name (genome } \\
\text { group) }\end{array}$} & \multirow[t]{2}{*}{$\begin{array}{l}\text { Pulp color } \\
\text { At use }\end{array}$} & $\begin{array}{l}\text { Harvest+ } \\
\text { color Strip } \\
\text {-carotene }\end{array}$ & \multicolumn{3}{|c|}{$\begin{array}{c}\text { Number of household indicating } \\
\text { choice }\end{array}$} \\
\hline & & \multicolumn{4}{|c|}{ South Kivu Region } \\
\hline & & 7121214 & $143(68$ & & \\
\hline & & & & & \\
\hline & & & & & \\
\hline & & $9 / 21355 \mathrm{U}$ & $27(13$ & & \\
\hline \multicolumn{6}{|c|}{ North Kivu Region } \\
\hline & & $7 / 2121 U$ & & & \\
\hline & & & & & \\
\hline & & & & & \\
\hline lusilc & ge & $9 / 37$ & 7\%) & $6 \%)$ & (2) \\
\hline
\end{tabular}

\section{Popular Cooking and Processing Methods of the Preferred Musa Varieties Practiced by Households in Eastern DRC}

It was established that the processing techniques applied to the Musa cultivars in North Kivu were similar to those in South Kivu [Table-4]. The two most popular EAHB cultivars ('Nshikazi' and 'Vulambya') were mostly utilized between stages 1 and 3 of ripening, i.e., before the fruit was ripe [15]. The fruits were generally peeled and boiled with only the addition of salt. Occasionally locally processed palm oil was added during the boiling process. The cooked banana was eaten either alone or with boiled beans or peas. In some instances the banana would be boiled together with beans and some amaranth leaves, 'lengalenga'. The plantain cultivars ('Musheba' and 'Musilongo') were also boiled but generally no salt was added because of the cultivars' natural sweetness and were eaten either alone or served with boiled green peas 'Musheba' and 'Musilongo' were also used to make 'Nchimba' banana flour. To make 'Nchimba', the fruit was harvested at maturity, kept for about 1 week to reach stage 5 of ripening, all yellow with green tips [15], peeled, chopped into pieces, sun-dried on the ground and either pounded or milled into flour. This flour (Nchimba) was then used to make porridge, a weaning food. In some cases, cassava flour was added to the plantain flour at a ratio of 2:3. Occasionally, the plantain cultivars were deep fried in the local palm oil and eaten as 'banana fries'.

Table 4- Local cooking/processing methods applied to common Musa cultivars in Eastern Democratic Republic of Congo

\begin{tabular}{|c|c|c|c|}
\hline Musa cultivar & Cooking method & $\begin{array}{l}\text { Cooking } \\
\text { ingredients }\end{array}$ & $\begin{array}{l}\text { Common } \\
\text { accompaniments }\end{array}$ \\
\hline \multirow[t]{2}{*}{ Nshiké } & Boiling ('kitika') & Salt, water & $\begin{array}{l}\text { Beans, peas, } \\
\text { amaranths, }\end{array}$ \\
\hline & Steamir & Salt, water, & Beans, meat ${ }^{2}$, Fish ${ }^{2}$ \\
\hline \multirow[t]{2}{*}{$\begin{array}{l}\text { Vulambya } \\
\text { (AAA) }\end{array}$} & $\begin{array}{c}\text { Boilin } \\
\text { ('kisamunyu') }\end{array}$ & Salt and water & $\begin{array}{l}\text { Beans, peas, ama- } \\
\text { ranths, }\end{array}$ \\
\hline & Boiling ('ndizi') & W & Peas, meat ${ }^{2}$, chicken ${ }^{2}$ \\
\hline \multirow{3}{*}{$\begin{array}{l}\text { Musheba } \\
\text { (AAB) }\end{array}$} & Deep frying ${ }^{3}$ & Palm oil & Meat $^{2}$, chicken ${ }^{2}$ \\
\hline & Roasti & . & \\
\hline & Porr & Water, cassava flour ${ }^{1}$ & - \\
\hline \multirow{3}{*}{$\begin{array}{l}\text { Musilongo } \\
\text { (AAB) }\end{array}$} & Boiling ('ndizi') & Water & Peas, meat ${ }^{2}$, chicken ${ }^{2}$ \\
\hline & Deep frying ${ }^{3}$ & & Mea \\
\hline & Porridge (flour) & Water, cassava flour ${ }^{1}$ & - \\
\hline
\end{tabular}

1 = Optional, 2 = Used occasionally, 3 = Prepared occasionally

\section{Consumption Pattern of Musa Fruit}

A 24-hour diet recall on all the food that had been consumed by the households indicated that $64-67 \%$ of all respondents had consumed EAHB cooking varieties within the last $24 \mathrm{hrs}$. (no significant difference between SK and NK) [Table-5]. The consumption of plantain cultivars was $4.8 \%$ and $10 \%$ in SK and NK, respectively. The majority of plantains, however, were not domestically consumed, but instead sold for cash due to their relatively high market price. Among cooking bananas, plantain bananas and desert bananas, cooking bananas (AAA-EAHB) were the most popular while desert bananas were the least popular [Fig-2] in both NK and SK regions.

Table 5- Consumption of cooking bananas and plantains in relation to other starchy staples available to communities in Eastern Democratic Republic of Congo

\begin{tabular}{lcccc} 
Starchy staples & \multicolumn{2}{c}{ South Kivu N= 208 } & \multicolumn{2}{c}{ North Kivu N=163 } \\
& Frequency $(\%)$ & S.E.Mean & Frequency $(\%)$ & S.E.Mean \\
Millet & 0.5 & $( \pm 0.005)$ & 0 & $( \pm 0.000)$ \\
Sorghum & 5.8 & $( \pm 0.016)$ & 0.6 & $( \pm 0.006)$ \\
Maize & 17.8 & $( \pm 0.027)$ & 14.2 & $( \pm 0.028)$ \\
Wheat \& products & 0 & $( \pm 0.000)$ & 3.1 & $( \pm 0.014)$ \\
Rice & 1 & $( \pm 0.007)$ & 1.8 & $( \pm 0.011)$ \\
Irish potatoes & 0.5 & $( \pm 0.005)$ & 0.6 & $( \pm 0.006)$ \\
Sweet potatoes & 0.5 & $( \pm 0.005)$ & 0 & $( \pm 0.000)$ \\
Cassava \& products & 86.6 & $( \pm 0.026)$ & 99.4 & $( \pm 0.006)$ \\
Yam/Taro & 0 & $( \pm 0.000)$ & 1.2 & $( \pm 0.009)$ \\
Cooking banana & 66.5 & $( \pm 0.034)$ & 64 & $( \pm 0.038)$ \\
Plantain & 4.8 & $( \pm 0.015)$ & 10 & $( \pm 0.021)$ \\
\hline
\end{tabular}

Boiling was the most popular Musa fruit processing method in both regions [Table-6]. In SK, $75 \%$ of respondents consumed boiled bananas while in NK the figure was $69 \%$. The varieties most used were the popular AAA-EAHB cultivars ('Nshikazi' in SK and 'Vulambya' in NK), which were boiled at ripening stages 1, 2 or 3 
(when the mature fruits were still green). Although a significant amount of roasted banana was consumed in SK (47 \%), none of the households in NK consumed the fruits this way. Other products were banana juice, beer and porridge in SK, and banana beer in NK. The beer banana cultivars were the AAA-EAHB 'Nshikazi' cultivar in SK and the AB 'Kisubi' cultivar in NK, which were kept up to ripening stage 6 (all yellow) before use [Table-6].

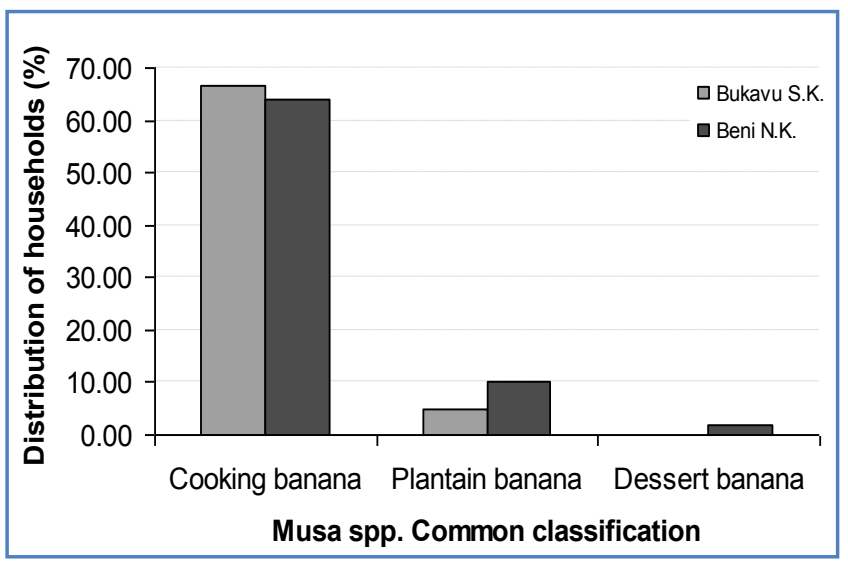

Fig. 2- Consumption rates of cooking, plantain and dessert bananas among households in Eastern Democratic Republic of Congo (SK-South Kivu: N=208, NK-North Kivu: N=163)

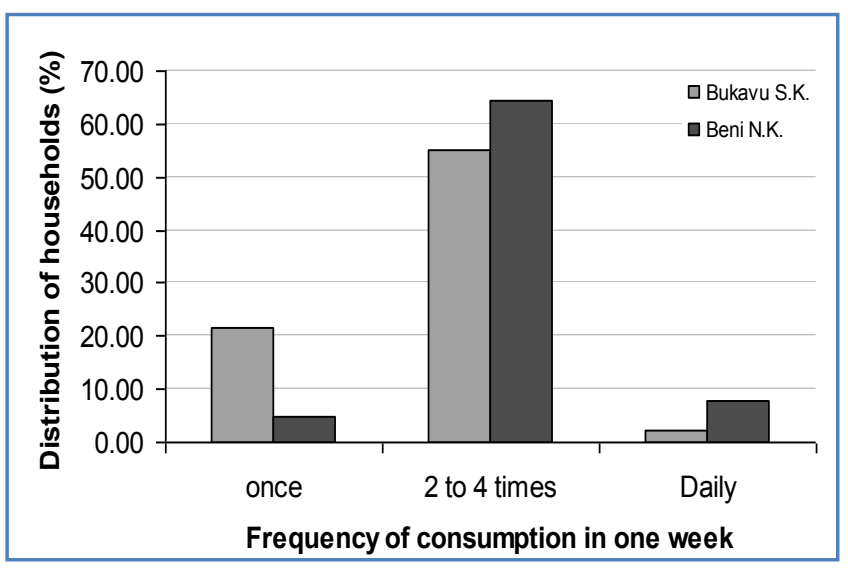

Fig. 3- Consumption rate of bananas and banana products among households in Eastern Democratic Republic of Congo (SK-South Kivu: N=208, NK-North Kivu: $N=163$ )

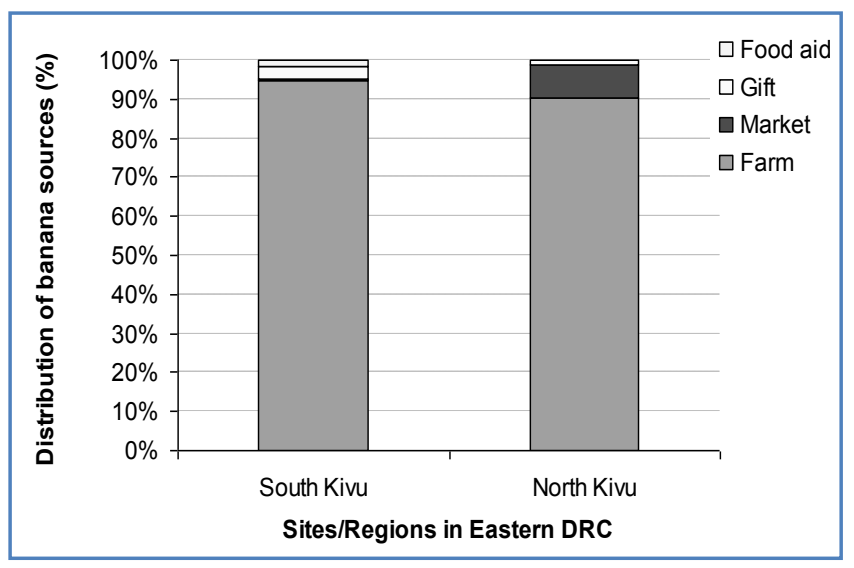

Fig. 4- Sources of bananas and plantains consumed by households in Eastern Democratic Republic of Congo (SK-South Kivu: $\mathrm{N}=208$, NK-North Kivu: $\mathrm{N}=163$ )
Table 6- Consumption of local Musa products among households in South Kivu ( $N=208)$, North Kivu ( $N=163)$, Eastern Democratic Republic of Congo.

\begin{tabular}{|c|c|c|c|c|c|}
\hline Musa product & $\begin{array}{l}\text { Consumption } \\
\text { freq. }(\%)\end{array}$ & $\begin{array}{l}\text { S.E. } \\
\text { Mean }\end{array}$ & $\begin{array}{l}\text { Cultivars } \\
\text { commonly } \\
\text { used }\end{array}$ & $\begin{array}{l}\text { Genomic } \\
\text { group }\end{array}$ & $\begin{array}{c}\text { Ripening } \\
\text { stage at } \\
\text { use }\end{array}$ \\
\hline \multicolumn{6}{|c|}{ Region/ Site South Kivu } \\
\hline Steamed banana & 1.2 & $( \pm 0.00)$ & $\begin{array}{c}\text { Nshikazi, } \\
\text { Barhebesha }\end{array}$ & AAA & 3 (un \\
\hline Boiled & 75 & & $\begin{array}{l}\text { Nshikazi, } \\
\text { Barhebesha }\end{array}$ & AAA & -3 (un \\
\hline Roast & 47 & $( \pm 0.035)$ & $\begin{array}{l}\text { Nshikazi, } \\
\text { Barhebesha }\end{array}$ & AAA & 1-3(unripe \\
\hline$S$ & 0 & $( \pm 0.000)$ & & $A A$ & 5 (ripe) \\
\hline & 6 & 0) & & & 6(ripe) \\
\hline & 13 & $0.000)$ & & & 6(ripe) \\
\hline & 4 & $( \pm 0.014)$ & Mush & A & 5 (ripe) \\
\hline \multicolumn{6}{|c|}{ Region/ Site North Kivu } \\
\hline ean & 0 & $( \pm 0.000)$ & & & \\
\hline & 69 & & Vulam & AAA & -3(unri \\
\hline & 0 & $( \pm 0.000)$ & 年 & 年 & \\
\hline sps & 0 & $( \pm 0.0000$ & Musilongo & $A A B$ & 5 (ripe) \\
\hline & 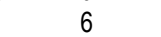 & $( \pm 0.019)$ & Kisul & $A B$ & 6(ripe) \\
\hline & 1 & $( \pm 0.0$ & & & 6(ripe) \\
\hline anana porridge & 1 & $( \pm 0.006)$ & Musilongo & $A A B$ & 5(ripe) \\
\hline
\end{tabular}

A large proportion of the household members (>55\%) from both NK and SK had consumed Musa fruit (bananas and plantains) between 2 to 4 times in the 7 days before the interview and there were some households that had consumed Musa fruit every day of the last 7 days preceding the survey [Fig-3]. Although there were several geographic sources of food for the community, more than $90 \%$ of the households from NK and SK indicated having obtained the bananas and plantains from their farms [Fig-4].

\section{Discussion}

\section{Demographic Data}

FAO studies confirm that rural women make a tremendous contribution to food and agricultural production. They also play a crucial role in determining and guaranteeing food security and well-being for the entire household [13]. It was, therefore, necessary that questions requiring details on household consumption patterns were addressed to women (caregivers). This explains the high percentage of women respondents $(91.7 \%)$. The majority of the women interviewed were between 25 - 35 years old, with $20 \%$ of them between the ages of 15 and 24 years, indicating that the women get married or become caregivers at a very early age. In $\mathrm{DRC}$, the legal minimum age for marriage is 15 for women and 18 for men, and $74 \%$ of women between 15 and 19 years of age are already married [16]. One of the most common outcomes of marriage at an early age is the withdrawal of the girl from formal education, or no school enrollment at all. This could explain the low level of education observed in this study, which puts these women at a disadvantage since educated women are more likely to feel empowered to influence decisions affecting food choices, availability and accessibility [17]. In addition, the low level of education reduces the ability of these women to process information, acquire skills and model positive care giving practices to improve health, nutrition and education outcomes for their children [17]. The low literacy level could further be explained by the irregularity and inadequacy of teachers' salary payments and inadequate infrastructure (negatively affecting teaching morale) [2]. The reported absence of primary education fee waivers [2] and the observed low monthly 
average income (<US\$30), could also be a contributor to high illiteracy. The girls may be forced to drop out of school due to a lack of money for tuition fees. The low income level observed in this study is also supported by a report which indicated that more than $80 \%$ of Congolese live on less than $\$ 1$ per day [13].

\section{Most Popular and Preferred Musa Cultivars}

Findings indicated that an average of 16 and 19 Musa cultivars were being cultivated by the communities in SK and NK, respectively. This number was lower than that of a total of 29 and $32 \mathrm{Mu}$ sa cultivars reported as being cultivated in SK and NK, respectively [18]. Both results show, however, that the diversity of Musa cultivars is slightly higher in NK as compared to SK, the higher number of cultivars reported in the earlier study could be due to the survey methodology used, where direct observation (transect walks) were carried out to establish the varied cultivars [18]. In addition more territories were included in the study as opposed to this study, where information was only collected through the FGDs and household interviews and only one territory/district from each of the two provinces (South Kivu and North Kivu) was included. In addition, not all villages were visited from the selected territories.

Although a beer banana cultivar 'Pisang Awak' (ABB), known locally as 'Kayinja' was not among the first four popular cultivars and did not even emerge in the preferred list, a study carried out in 2005 cites 'Kayinja' as the most popular cultivar occupying nearly $3 / 4$ of the land cropped with banana in Eastern DRC [19]. This could be because the participants of the FGDs were mostly local farmers and women of child-bearing age, and they seemed to prefer cultivars that could be used to make food. The most popular cultivars identified included three cooking cultivars and one plantain. According to the community members, 'Nshikazi'(AAA-EAHB) has high yield (big bunch) and good cooking quality. These findings confirm findings by [18], carried out in the same province (SK) although at different districts which also cited 'Nshikazi' as the most preferred Musa cultivar. According to [18], Nshikazi' was occupying $52 \%$ of the land under banana and apart from having the traits already mentioned (high yield, high market price and good cooking quality), 'Nshikazi' tastes better after cooking, and made good banana juice and banana beer. 'Nshikazi' is also grown in Rwanda where it is known as 'Ishika' and Uganda where it has different names depending on the regions i.e. 'Ensika', 'Emburansika' 'Omuburasika' or 'Nsika' [Table-1]. 'Musheba', a plantain cultivar whose name also means 'wedding' [Table-1], was the most popular plantain cultivar in SK. Apart from the reasons given by the community (high market price, good yield, and good cooking quality), other studies have reported that 'Musheba' is a popular cultivar because it matures faster and tastes very good [18]. In addition, 'Musheba' has social-cultural importance in times of marriage and the 'Mashi' tribe of SK had obtained it from Uganda as a form of dowry [18].

In North Kivu, the findings showed that the four most popular Musa cultivars were 'Vulambya', 'Kiware', 'Nguma' and 'Musilongo'. These four cultivars were also among the six most preferred Musa cultivars in NK identified in a previous study within the same region [18]. The reasons for Vulambya's popularity were similar to those of 'Nshikazi' in SK. Although 'Nguma' has been reported to be most popular cultivar in NK and 'Musilongo' didn't appear in the ten most popular cultivars [18], the findings of this study indicated that both 'Nguma' and 'Musilongo' were popular. In addition, the community members indicated that 'Musilongo' produces a bigger bunch and matures faster than 'Nguma'.

Among the above preferred plantains and cooking banana cultivars, the pulp color of the plantain cultivars was darker (orange) while the pulp color of the cooking banana varieties was lighter (yellow and ivory). These results are similar to those observed after pre-screening of cultivars from Uganda, Cambodia, Philippines and Hawaii, which revealed that more than $80 \%$ of the plantain cultivars (AAB) screened had an orange pulp while more than $80 \%$ of the cooking banana cultivars (AAA) had pulp color ranging from ivory to white [1]. Although the pulp color is not a definite indication of levels of carotenoids, it helps in filtering cultivars that could be further analyzed for enhanced carotenoids content. If a variety is found to contain considerable amounts of carotenoids, it could be used for fast-tracking approaches aimed at reducing VAD among Musa dependent rural households of Eastern DRC.

\section{Popular Musa Cooking and Processing Methods Practiced by Households in Eastern DRC}

As indicated earlier, in Eastern DRC bananas are a major part of the staple diet, complementing other sources of food. The methods of processing and cooking banana fruit range from simple boiling to fermentation, drying and grinding to make flour; The fruit can also be eaten as a dessert, or processed into juice, beer and wine [20]. The purpose of processing/cooking is to make the fruit more palatable and digestible. Processing also extends the storage life of the fruit, which is often highly perishable in its fresh condition. Findings of this study indicate that in both NK and SK, the bananas (cooking banana and plantains) were mostly boiled. Other cooking methods practiced included deep frying the plantains in local palm oil, but this was not so common since most of the harvested plantain bananas were sold to urban dwellers to generate income. Boiling of the banana fruit is also a common practice in Uganda, Cameroon, Burundi and West Indies. In Uganda, the fruit is boiled with beans and ghee then mixed with pepper, salt and onions to make a dish called 'akatogo'. In Cameroon, the green banana is boiled and served in a sauce of palm oil with fish, cooked meat, green beans and seasoning, whereas in the West Indies boiled green banana is served with salted fish or meat [21].

As noted, apart from boiling the plantain varieties were also harvested dried and ground into flour mostly used for making porridge for children less than 5 years old. According to the community members the porridge made from plantain flour is especially useful for children with diarrhea. It was also indicated that the banana flour could be mixed with cassava flour and not only used for making baby porridge but also boiled in water to make a thick paste locally called 'ugali.' The 'ugali' could be consumed with any relish, the most common one being, boiled cassava leaves, locally called 'sombe'. Musa flour especially from plantain banana was the only local Musa product that seemed to have a long shelf life. The same is true of practices in Gabon and Cameroon, where the mature green form of banana is dried and stored and may be used for cooking after grinding into flour, the difference is that in these two countries (Gabon and Cameroon) this product was mainly used as a famine reserve, not an everyday meal [21]. Steaming and roast- 
ing are very popular banana cooking methods in Uganda; the EAHB cultivars are mostly steamed in banana leaves and either pounded or eaten whole (a dish called 'matoke'), while the plantains are roasted and sold as street food. Despite this, findings of this study showed that these two cooking methods (steaming and roasting) are not popular in Eastern DRC.

\section{Musa Fruit Consumption Patterns}

Of all the staples considered in this study, cassava root was the most highly consumed. This finding is supported by the high cassava production of $74 \%$ observed by other researchers [2]. These researchers also indicated that DRC has the highest annual cassava consumption in the world, with an estimated $390 \mathrm{~kg}$ fresh root (equal to $1,100 \mathrm{kcal}$ ) per person per day[2]. Cassava is cultivated in around $50 \%$ of the arable land in Congo and provides to $70 \%$ of Congolese population $60 \%$ of food energy intake [2]. Just as reported [2], findings of this study showed that EAHBs were the second most consumed starchy staple recording a consumption rate of $\geq 70 \%$ among households in both SK and NK [Table-5] and [Fig-2]. Other researchers have also reported a EAHBs consumption rate of almost $80 \%$ in DRC [22]. Although the consumption of plantains has also been reported to be about $36 \%$ in SK and that plantains have traditionally been a starchy staple food of rural populations in the humid lowlands of DRC [22], in this study, the low level of plantain consumption observed (4.8\% SK and $10 \%$ NK) could be explained by their current $r$ high market value, thus farmers are increasingly selling plantains as a cash crop to urban consumers. With rapid urbanization and the growing prosperity of city dwellers, demand is outstripping supply, thereby leaving the rural poor households to depend on either cooking varieties or other starchy staples. The consumption of dessert bananas was even lower with consumption rates of $0 \%$ and $2 \%$ in SK and NK, respectively [Fig2], because dessert bananas are mostly consumed as snacks and their production is also very low in Eastern DRC.

More than $90 \%$ of the households from both NK and SK obtained the bananas from their farms [Fig-2]. This is higher than values reported earlier [22], where only $64 \%$ of households in SK obtained their bananas from own production and $24 \%$ relied on purchase. This difference could be because in the reported study [22], the respondents included both rural and urban traders in Musa while in this study the respondents were exclusively small-holder farmers.

\section{Conclusions and Recommendations}

Bananas (cooking banana and plantains) are the second most popular starchy staple consumed by smallholder households in SK and NK of Eastern DRC. 'Nshikazi' (AAA-EAHB) and 'Musheba' (AAB-French plantain) are the most popular and preferred Musa cultivars in South Kivu while 'Vulambya' (AAA-EAHB) and 'Musilongo' (AAB-French plantain) are the most popular and preferred Musa cultivars in North Kivu. The AAA-EAHB cultivars ('Vulambya' and 'Nshikazi') are preferred because they produce big bunches, posses good cooking qualities and they make good banana beer. The plantain cultivars ('Musheba' and 'Musilongo') are preferred because they mature faster and have high market prices.

The fruit pulp color of the preferred Musa cultivars ranges from yellow to orange, an indication of potentially high carotenoids levels. A nutritional analysis should be carried out to verify whether they could indeed be a potential source of carotenoids. Of the three common Musa fruit classifications (cooking banana, plantain banana and desert banana), cooking bananas are the most consumed, having a consumption rate of more than $65 \%$ in both North Kivu and South Kivu.

Although the Musa fruit is normally processed/cooked using various methods such as boiling (with/without peel), steaming, roasting, drying and milling into flour, the most popular cooking method applied to the Musa cultivars consumed in Eastern DRC is boiling without peel. More than $50 \%$ of the households consume boiled bananas between two and four times per week, with $20 \%$ of the households consuming it on daily basis with $90 \%$ of the bananas obtained from the household's farm.

In the light of these results, it is recommended that thenutrition content of these preferred cultivars be examined to establish whether, the Musa fruits could be a good source of other micronutrients, especially provitamin A carotenoids. In addition, the Musa fruit is not consumed immediately after harvest, and in most cases, it is cooked into various products. Although maturation or ripening in Musa fruits after harvest is usually accompanied by enhanced carotenogenesis [23], some preliminary studies have shown that the impact of ripening is cultivar-specific, with pVACs contents remaining essentially unaltered, increasing or even slightly decreasing depending on the genotype [6].

Several reports have shown evidence that although results are still conflicting, retention or loss of carotenoids during storage/ripening and processing of Musa fruit is cultivar dependent [24, 23]. There is need to therefore carry out more research that will include a variety of Musa cultivars from Eastern and Central African to ascertain the content of carotenoids in these fruits, the stage in fruit development where carotenoid levels reach their peak and processing/cooking techniques that retain or avail more carotenoids. If the levels of carotenoids are found to be high in some of these local Musa cultivars, they could be used in the fast-tracking approach, to reduce Vitamin A deficiency among Musa-dependent populations of Eastern and Central Africa.

\section{Acknowledgement}

The authors acknowledge the Consortium for Improving Agriculture -based Livelihoods in Central Africa (CIALCA) for providing the necessary funding to carry out this study. Sincere gratitude goes to the team of persons who facilitated the data collection from the study areas. These include Mr. Charles Lwanga and Professor Ndung'o Vigheri of North Kivu, Beni territory, Mr. Jules Ntamwira and Mr. Charles Bisimwa of South Kivu, Bukavu territory, the enumerators and households" members from both NK and SK. Mr. Johnson Vincent science editor, Bioversity International in Montpellier- France is thanked for his assistance in editing this paper

\section{References}

[1] HarvestPlus (2007) Annual Technical Report.

[2] Luntala T., Mbile F., Jean Claude O. and M. Okulo (2000) Country Presentation, DRC.

[3] Tenkouano A., Vuylsteke D. and R. Swennen R. (2007) Journal of Crop Science Improvement, 20(1-2), 31-51.

[4] Stover R.H. and Simmonds N.W. (1991) Bananas, Tropical 
Agriculture Series, 3rd ed., London, 468.

[5] Davey M.W., Keulemans J. and Swennen R. (2006) Science Direct Journal of Chromatography, A(1136), 176-184.

[6] Davey M.W., Van den Berge I., Markham R., Swennen R. and Johan K. (2009) Journal of Food Chemistry, 115, 806-813.

[7] Sub Committee on Nutrition (2010) Sixth Report on the World Nutrition Situation, Geneva, 2, 8-22.

[8] Mulokozi G.I. (2003) Content and In vitro accessibility of Provitamin A carotenoids in some Tanzanian Vegetables and fruits. PHD Thesis, Chalmers University of Technology, Goteborg, Sweden.

[9] Ministry of Planning (2005) Piloting Unit of DSRP Process, Kinshasa-Gombe, DRC, 129-155.

[10]World Food Program (2009) Fighting Hunger World Wide. Democratic Republic of Congo.

[11]World Food Program (2008) Comprehensive Food Security and Vulnerability Analysis, Executive Brief: Democratic Republic of Congo.

[12]Magnani R. (1997) IMPACT Food Security and Nutrition Monitoring Project, Arlington, Va.

[13]FAO (2003) Food Security Responses to the Protracted Crisis Context of the Democratic Republic of Congo.

[14]Dadzie B.K. and Orchard J.E. (1997) Criteria and Methods, INIBAP Technical Guidelines, 2, 63.

[15]Ngoh G.N., Tomekpe K., Fokou E. and Etoa F.X. (2009) Global Science Books, 3(1), 64-70.

[16]FIDH (2010) Democratic Republic of the Congo: Breaking Impunity.

[17]ACC/SCN (2004) 5th Report on the Worlds' Nutrition Situation, Geneva, 5-14.

[18]Dowiya N.B., Rweyemamu C.L. and Maerere A.P. (2009) Journal of Animal \& Plant Sciences, 4(2), 341-356.

[19]Ndungo V., Bakelana K., Eden-Green S. and Blomme G. (2005) Infomusa, 13, 43-44.

[20]Ndungo V., Fiaboe K.K.M. and Mwangi M. (2008) Journal of Applied Bioscience, 1(1), 1-7.

[21]FAO (1990) FAO Corporate Document Repository.

[22] Jagwe J., Ouma E., Van Asten P. and Abele S. (2008) CIALCA Project Survey Report, Bujumbura, 61.

[23]Ngoh G.N., Lusty C., Van den Bergh I., Akyeampong E., Davey M.W. and Tomekpe K. (2008) Global Science Books, 2(2).

[24]Lusty C., Akyeampong E., Davey M.W., Ngoh Newilah G. and Markham R. (2006) InfoMusa, 15(1-2). 